\title{
Phase-weighted UWB Imaging through Huygens Principle
}

\author{
Gianluigi Tiberi ${ }^{1}$, Banafsheh Khalesi ${ }^{1}$, Behnaz Sohani $^{1}$, Navid Ghavami ${ }^{2}$, \\ Sandra Dudley ${ }^{1}$, and Mohammad Ghavami ${ }^{1}$ \\ ${ }^{1}$ School of Engineering, London South Bank University, London, UK \\ ${ }^{2}$ UBT - Umbria Bioengineering Technologies, Spin off of University of Perugia, Perugia, Italy
}

\begin{abstract}
Phase information is used in many imaging modalities to enhance resolution, such as in magnetic resonance imaging. However, its impact in Ultra-wideband (UWB) microwave imaging is still unexplored. Here, examples using analytical simulations are shown to address the impact of phase information in Huygens principle (HP) based UWB imaging. Conventional amplitude-weighted HP imaging has a resolution, which achieves the optical resolution limit of $\lambda / 4$, where $\lambda$ represents the wavelength in the investigation domain calculated at the highest frequency. Simulation results show that HP phase-weighted modality leads to an improvement in the resolution of approximately $30 \%$ with respect to the HP amplitude-weighted modality, and to an increase in signal-to-average ratio of approximately $40 \%$ with respect to the HP amplitudeweighted modality.
\end{abstract}

\section{INTRODUCTION}

Ultra Wide Band (UWB) imaging has attracted growing attention, especially for its applicability to breast cancer detection, motivated by the significant contrast in the dielectric properties of normal and malignant tissues at microwave frequencies [1]. Recently, UWB imaging has also been applied to bone imaging [2], human forearm imaging [3] and skin cancer detection [4]. Current research in UWB imaging can be divided mainly into tomography and linear scattering techniques. Among linear scattering techniques, the Huygens principle (HP) based technique allows us to detect dielectric inhomogeneities in the frequency domain [5]. Specifically, using HP to forward propagate the UWB signals collected outside the investigation domain permits the capture of contrast, i.e., the extent to which different tissues, or different condition of tissues, can be discriminated in the final image. It has been shown [5] that HP resolution achieves the optical resolution limit of $\lambda / 4$, where $\lambda$ represents the wavelength in the investigation domain calculated at the highest frequency. However, the impact of phase information should be still investigated.

Phase information is used in many imaging modalities to enhance resolution (such as in Magnetic Resonance Imaging [6]). Here, examples using analytically based simulations are shown to address the impact of phase information in imaging parameters such as resolution and signal-to-average ratio. In this context, with the aim of mimicking a bone fracture, a $0.5 \mathrm{~mm}$ radius cylinder having the dielectric properties of blood/muscle has been positioned inside a $6 \mathrm{~cm}$ radius cylinder having the dielectric properties of bone cortical.

\section{THEORY}

Let us consider the problem depicted in Fig. 1, where a cylindrical phantom having radius $a_{0}$, dielectric constant $\varepsilon_{r 1}$ and conductivity $\sigma_{1}$, is illuminated by a transmitting line source $t x_{m}$ located at $\left(a_{t x}, \phi_{m}\right)$. The transmitting source operates in air at a given frequency $f$. The phantom may contain an inclusion (not shown in Fig. 1) with a different dielectric constant and/or conductivity, denoted as $\varepsilon_{r 2}$ and $\sigma_{2}$ respectively. The problem consists of identifying the presence of the inclusion by using the field received at many points displaced all around the phantom. Thus, suppose the (complex) electric field at the points $r x_{n p} \equiv\left(a_{0}, \phi_{n p}\right) \equiv \vec{\rho}_{n p}$ is known, that is:

$$
S_{21 n p}^{m}\left(a_{0}, \phi_{n p} ; t x_{m} ; f\right)
$$

where $n p=1,2, \ldots N_{P T}$ indicates the receiving points; $m$ indicates the transmitting source, and $f$ is the frequency. The received signals are then processed through HP to calculate the field inside the cylinder [5]. Specifically, we have:

$$
E_{H P, 2 D}^{r c s t r}\left(\rho, \phi ; t x_{m} ; f\right)=\sum_{n p=1}^{N_{P T}} S_{21 n p}^{m}\left(a_{0}, \phi_{n p} ; t x_{m} ; f\right) G\left(k_{1}\left|\vec{\rho}_{n p}-\vec{\rho}\right|\right)
$$


with $G\left(k_{1}\left|\vec{\rho}_{n p}-\vec{\rho}\right|\right)$ representing the Greens function and $(\rho, \phi) \equiv \vec{\rho}$ representing the observation point, while $k_{1}$ indicates the wave number for the media constituting the external cylinder, here assumed to be known. However, it has been shown that detection can also be achieved if using the free space wave number [7].

In (??), the string rcstr is used to indicate the "reconstructed" internal field, while the string HP indicates that a HP based procedure is employed. The reconstructed field depends on the illuminating source and on the frequency $f$ (since the field at the points $r x_{n p} \equiv\left(a_{0}, \phi_{n p}\right) \equiv \vec{\rho}_{n p}$ depends on these parameters). Assuming we use NF frequencies $f_{i}$ in the band $B$, we can write:

$$
\begin{aligned}
I_{M A G}\left(\rho, \phi ; t x_{m}\right) & =\frac{\Delta_{f}}{B} \sum_{i=1}^{N_{F}}\left|E_{H P}^{r c s t r}\left(\rho, \phi ; t x_{m} ; f_{i}\right)\right| \\
I_{P H}\left(\rho, \phi ; t x_{m}\right) & =\frac{\Delta_{f}}{B} \sum_{i=1}^{N_{F}}\left|\operatorname{angle}\left(E_{H P}^{r c s t r}\left(\rho, \phi ; t x_{m} ; f_{i}\right)\right)\right|
\end{aligned}
$$

where $I_{M A G}$ is the amplitude-weighted intensity and $I_{P H}$ is the phase-weighted intensity. In (??)$(? ?), \Delta_{f}$ is the frequency step.

To remove the artefacts, we employ the subtraction between $S_{21}$ obtained using two transmitting line sources slightly displaced, i.e., $5^{\circ}$, along the azimuth [8].

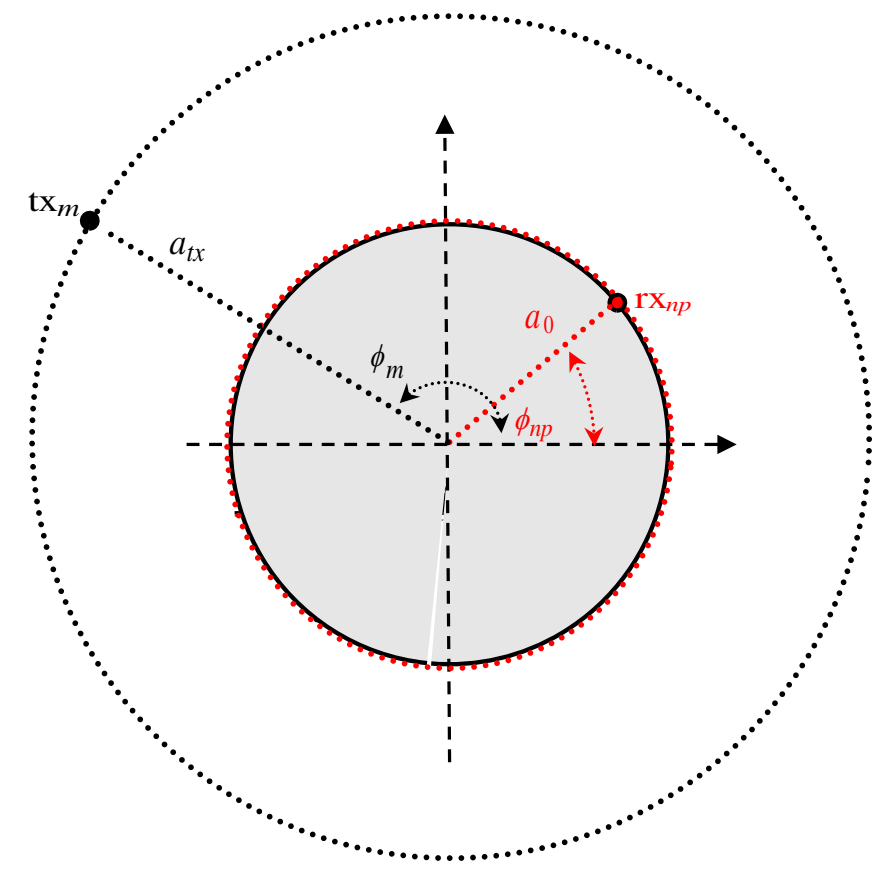

Figure 1. Pictorial view of the problem.

\section{RESULTS}

The capability and performances of the amplitude-weighted and the phase-weighted intensity to detect an inclusion has been verified here through simulations.

In particular, we consider a cylinder in free space with radius $a_{0}=6 \mathrm{~cm}, \varepsilon_{r 1}=10$ and $\sigma_{1}=$ $0.5 \mathrm{~S} / \mathrm{m}$. The cylinder has an eccentrically placed cylindrically shaped inclusion having radius $a_{1}=0.5 \mathrm{~mm}, \varepsilon_{r 2}=60$ and $\sigma_{2}=2 \mathrm{~S} / \mathrm{m}$, as summarized in Table 1 . The distance between the centres of the two cylinders is $2 \mathrm{~cm}$ and the phi offset is equal to $60^{\circ}$. The cylinder is illuminated by two transmitting line sources: $t x_{1}$ located at $\left(a_{t x}=20 \mathrm{~cm}, \phi_{1}=0^{\circ}\right)$ and $t x_{2}$ located at $\left(a_{t x}=20 \mathrm{~cm}\right.$, $\phi_{2}=5^{\circ}$ ). The band $1-5 \mathrm{GHz}$ is considered (slightly greater than that used in [2]) and a frequency sample spacing of $5 \mathrm{MHz}$ is used. For each illuminating source and for each frequency sample, the field at $N_{P T}=60$ points lying on the external surface is calculated analytically by resorting to [5].

Amplitude-weighted $(\mathrm{A}-\mathrm{W})$ and the phase-weighted $(\mathrm{P}-\mathrm{W})$ normalized linear intensity, calculated using (??) and (??) respectively, are shown in Fig. 2, after having performed artefact removal. 
In addition, A-W and P-W imaging performances are measured by suitable metrics such as resolution $(\mathrm{R})$ and signal-to-average ratio $(\mathrm{S} / \mathrm{A})$. Specifically: the resolution is the dimension of the region whose normalized intensity is above $1 / \sqrt{2} ; \mathrm{S} / \mathrm{A}$ ratio is maximum of the intensity divided by the average of the intensity. The aforementioned metrics are listed in Table 1.

Table 1. Geometrical and dielectric properties of the external cylinder and of the inclusion.

\begin{tabular}{|c|c|c|c|}
\hline & Radius $[\mathrm{mm}]$ & Dielectric constant & Conductivity $[\mathrm{S} / \mathrm{m}]$ \\
\hline External cylinder & 60 & 10 & 0.5 \\
\hline Inclusion & 0.5 & 60 & 2 \\
\hline
\end{tabular}

Table 2. Resolution (R) and signal-to-average ratio (S/A) calculated for the amplitude-weighted (A-W) and for the phase-weighted (P-W) normalized linear intensity.

\begin{tabular}{|c|c|c|}
\hline \multirow{2}{*}{} & \multicolumn{2}{|c|}{$1-5 \mathrm{GHz}$} \\
\cline { 2 - 3 } & $\mathrm{A}-\mathrm{W}$ & $\mathrm{P}-\mathrm{W}$ \\
\hline Resolution $[\mathrm{mm}]$ & $\sim 5$ & $\sim 3$ \\
\hline S/A (linear) & 6.5 & 9.2 \\
\hline
\end{tabular}

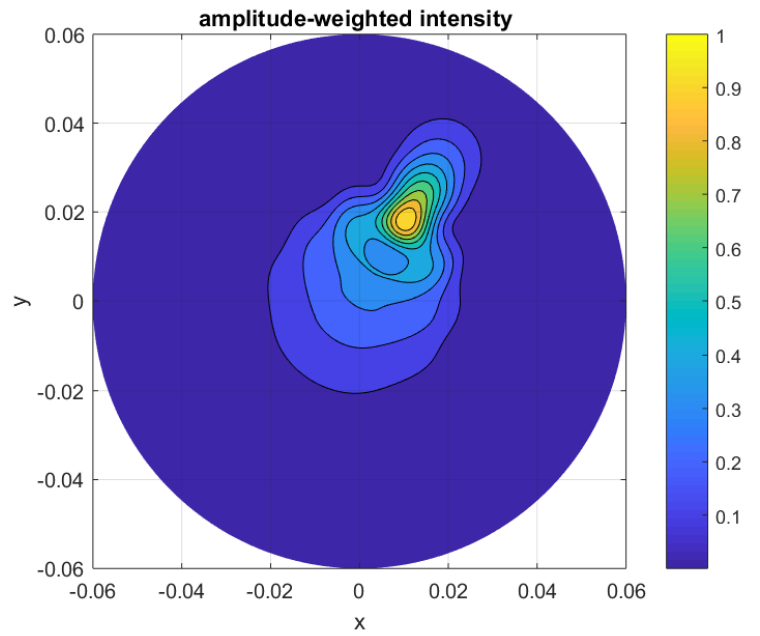

(a)

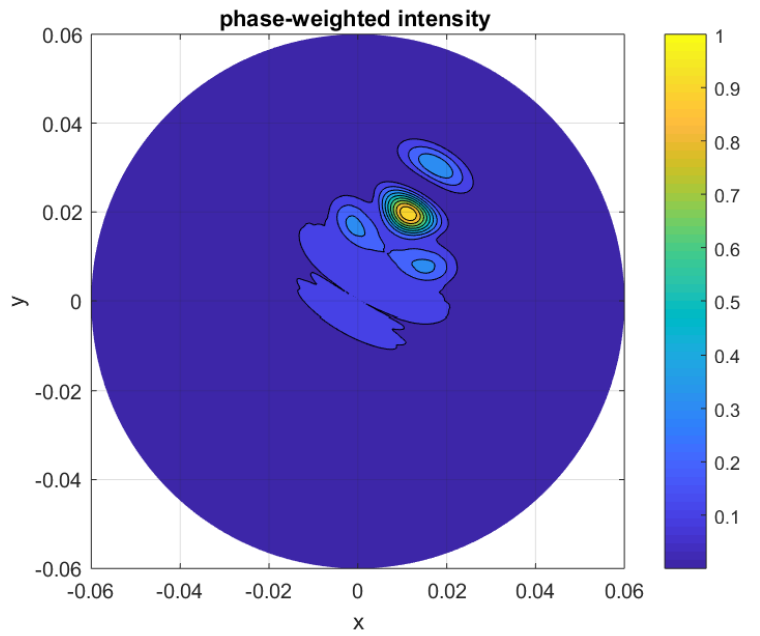

(b)

Figure 2. Amplitude-weighted (a) and the phase-weighted (b) normalized linear intensity obtained using Huygens Principle (HP)-based imaging procedure. UWB images are homogeneity maps of dielectric properties. $X$ and $Y$ are given in meters.

\section{DISCUSSIONS AND CONCLUSIONS}

Among non-coherent linear scattering techniques, the HP-based technique permits the detection of dielectric inhomogeneities in frequency domain. Employing HP for multi-bistatic configurations is suitable, and it can also be used if sources and phantoms are in free space, i.e., no coupling liquid is required.

Note that the dielectric property values of the example presented here were chosen in accordance with [9] to reproduce (at the central frequency) the dielectric properties of a bone cortical with a small fracture constituted of a mixture of blood/muscle. It is worthwhile pointing out that in the example presented here the electric properties of the cylinders have been assumed to be frequency independent; however, the frequency-dependence of the electric properties can also be taken into account by this approach.

Simulations shows that HP phase-weighted modality leads to an improvement in resolution of approximately $30 \%$ with respect to HP amplitude-weighted modality. In addition, phase-weighted modality may also lead to an improvement in signal-to-average ratio of approximately $40 \%$ with 
respect to HP amplitude-weighted modality. Such improvements in both resolution and signal-toaverage ratio may be fundamental towards the successful application of the procedure to in-vivo bone fracture imaging.

\section{ACKNOWLEDGMENT}

This project leading to this application has received funding from the European Union's Horizon 2020 research and innovation programme under the Marie Sklodowska-Curie grant agreement No. 793449.

\section{REFERENCES}

1. Nikolova, N. K., "Microwave imaging for breast cancer," IEEE Microwave Magazine, Vol. 12, No. 7, 78-94, 2011.

2. G. Ruvio, A. Cuccaro, R. Solimene, A. Brancaccio, B. Basile, and M. J. Ammann, "Microwave bone imaging: A preliminary scanning system for proof-of-concept," Healthcare Technology Letters, Vol. 3, No. 3, 218-221, 2016.

3. "Human forearms: Pilot study and image enhancement," International Journal of Biomedical Imaging, Vol. 2013, Article ID 673027, 17, 2013.

4. Ghavami, N., G. Tiberi, M. Ghavami, M. E. Lane, and S. Dudley, "Huygens principle based UWB Microwave imaging method for skin cancer detection," IEEE/IET International Symposium on Communication Systems, Networks and Digital Signal Processing (CSNDSP16), July 2016.

5. Ghavami, N., G. Tiberi, D. J. Edwards, and A. Monorchio, "UWB microwave imaging of objects with canonical shape," IEEE Transactions on Antennas and Propagation, Vol. 60, No. 1, 231-239, Publication Year: 2012.

6. Sedlacik, J., M. Barth, H.-J. Mentzel, J. R. Reichenbach, "Magnetic susceptibility-weighted MR phase imaging of the human brain alexander rauscher," American Journal of Neuroradiology, Vol. 26, No. 4, 736-742, Apr. 2005.

7. Ghavami, N., P. P. Smith, G. Tiberi, D. Edwards, and I. Craddock, "Non-iterative beamforming based on Huygens principle for multistatic ultrawide band radar: application to breast imaging," IET Microwaves, Antennas \& Propagation, Vol. 9, No. 12, 1233-1240, 2016.

8. Byrne, D. and I. J. Craddock, "Time-domain wideband adaptive beamforming for radar breast imaging," IEEE Transactions on Antennas and Propagation, Vol. 63, No. 4, 1725-1735, 2015.

9. Hasgall, P. A., F. Di Gennaro, C. Baumgartner, E. Neufeld, B. Lloyd, M. C. Gosselin, D. Payne, A. Klingenböck, and N. Kuster, "IT'IS Database for thermal and electromagnetic parameters of biological tissues," Version 4.0, May 15, 2018. 